\title{
Presencia de Quistes de Retención Mucoso del Seno Maxilar Detectados por Radiografías Panorámicas en Pacientes de la Ciudad de Temuco, Chile.
}

\author{
Presence of Mucus Retention Cysts in Maxillary Sinus Detected \\ by Panoramic Radiographs on Patients of Temuco, Chile.
}

"Ramón Fuentes Fernández; "Ivonne Garay Carrasco \& **Eduardo Borie Echevarría

FUENTES, F. R.; GARAY, C. I. \& BORIE, E. E. Presencia de quistes de retención mucosa del seno maxilar en radiografías panorámicas en pacientes de la ciudad de Temuco, Chile. Int. J. Morphol., 26(3):697-699, 2008.

RESUMEN: Los quistes de retención mucoso son una patología muy poco frecuente en el seno maxilar, perode fácil detección en radiografías panorámicas. El propósito de este estudio fue cuantificar los casos de mucoceles en senos maxilares durante un periodo de 5 meses, observando su frecuencia por edad y sexo. Se encontró una prevalencia de $2.06 \%$ en 339 radiografías revisadas, observándose mayor frecuencia en el género masculino y a una edad promedio de 30 años. Todos los casos no manifestaron sintomatología alguna.

PALABRAS CLAVE: Quistes de retención mucosa; Seno maxilar; Radiografías panorámicas.

\section{INTRODUCCIÓN}

El aumento del uso de la radiografía panorámica en la práctica odontológica ha detectado, por casualidad, anormalidades en el seno maxilar, especialmente de quistes de retención mucoso (Allard et al.,1981).

El quiste de retención mucoso del seno maxilar se origina por la acumulación de mucus en la línea de la mucosa sinusal como resultado de la obstrucción del ducto o glándula con su recubrimiento epitelial. Su crecimiento es lento, de características expansivas y produce reabsorción ósea. A nivel mundial se ha estimado una incidencia de 1.4-9.6\% de los pacientes (Ruprecht et al., 1986; Hadar et al., 2000; De Oliveira et al., 2004). Se presentan con mayor frecuencia en los senos frontal y etmoidal, en comparación con el seno esfenoidal y muy escasamente en el seno maxilar. El $10 \%$ de los mucoceles de los senos para-nasales se presenta en el seno maxilar. Se ha reportado mayor prevalencia en Japoneses y su incidencia en la tercera y cuarta década de la vida (De Oliveira et al.; Busaba \& Kieff, 2002.), observándose mayor prevalencia en el género masculino que en el femenino (Allard et al.). Poco más de la mitad de los quistes de retención mucoso se relacionan con algún tipo de reacción alérgica (Casamassimo \& Lilly, 1980). Los mucoceles del seno frontal son de crecimiento lento y localmente agresivos, causados por la pérdida de las propiedades de drenaje de la mucosa epitelial del seno. Pueden ser asintomáticos, pero también tienen propensión a expandir, remodelar y reabsorber las paredes óseas del seno comprometido alterando su integridad y, eventualmente, comprometiendo las estructuras circundantes como la órbita y la cavidad intracraneal, produciendo cefaleas, dolor periorbital o facial (Hadar et al.; De Oliveira et al.; Chiarini et al., 2000). El mucocele puede evolucionar, ocasionalmente, a un piocele. (Peric' et al., 2007).

Radiográficamente el quiste se observa como una figura redondeada, radiolúcida y generalmente se sitúa y se observa en el piso del seno (Casamassimo \& Lilly). Aproximadamente 6-23\% de los quistes del seno maxilar se rompen espontáneamente (Hadar et al.). Su etiología no está totalmente definida (Peric' et al.,) y se plantea que se puede producir por la obstrucción del ostio de drenaje del seno paranasal comprometido, como consecuencia de procesos crónicos de rinosinusitis, pólipos nasosinusales, trauma craneofacial, cirugías previas y tumores benignos o malignos, entre otros.

\footnotetext{
* Departamento de Odontología Integral, Universidad de La Frontera, Temuco Chile.

** Alumno Carrera Odontología, Universidad de La Frontera, Temuco Chile.
} 
La obstrucción del conducto nasopalatino es la causa más frecuente en la patogénesis de los mucoceles nasofrontales, pudiendo ser esta obstrucción adquirida o postraumática (De Oliveira et al.; Chiarini et al.).

El tratamiento es quirúrgico y solo se recomienda si la destrucción alrededor del hueso se ha presentado con sintomatología previa (Gothberg et al., 1976), siendo las vías de abordaje externa o endonasal las que con mayor frecuencia se utilizan. La endoscopía endonasal quirúrgica se ha utilizado recientemente para la marsupialización de los mucoceles paranasales, aún cuando es difícil de aplicar cuando el mucocele está situado en la parte anterior o lateral del seno maxilar (De Oliveira et al.; Shiomi et al., 2002). El presente estudio consistió establecer la presencia de mucoceles del seno maxilar en una muestra de radiografías panorámicas en la población de Temuco.

\section{MATERIAL Y MÉTODO}

Se revisaron 339 radiografías panorámicas obtenidas entre los meses de marzo y julio de 2008. Estas se realizaron mediante el uso de un ortopantomógrafo Proline CC Plan Meca. El proceso de revelado se efectuó en una reveladora Dürr X 24 Pro. Se utilizaron placas radiográficas Kodak T-Mat G/RA. Las radiografías fueron revisadas por un $\mathrm{Ci}$ rujano-Dentista Radiólogo, quien informó sobre la presencia de mucoceles en el seno maxilar. Los resultados se tabularon, se analizaron porcentualmente y se correlacionaron con variables etarias y de género.

\section{RESULTADOS}

Se encontraron 7 pacientes $(2.06 \%)$ del total de casos, que presentaban en sus radiografías quistes de retención mucoso del seno maxilar, todos sin haber manifestado a su Odontólogo de alguna sintomatología atribuible a los quistes encontrados. Correspondieron 6 casos $(85.7 \%)$ al sexo masculino y al sexo femenino solo un caso $(14,3 \%)$ presente en el lado izquierdo. En los hombres en 4 casos $(57,14 \%)$ el quiste se presentó en el lado derecho y en un caso $((28,58 \%)$ en el lado izquiero. Todos los casos se presentaron unilateralmente.

La presencia de quiste de retención mucoso se presentó en los hombres entre 16 y 55 años de edad y el único caso en una mujer de 41 años de edad.

\section{DISCUSIÓN}

A partir de los resultados se demuestra la importancia del examen radiográfico de los pacientes que acuden al Odontólogo a través de una radiografía panorámica por la posibilidad de poder encontrar diferentes patologías, en este caso quistes de los senos maxilares de retención mucoso (Allard et al.). Se encontró una prevalencia de $2.06 \%$ de pacientes que presentaron la alteración descrita, lo que es concordante con Ruprecht et al.; De Oliveira et al. y Busaba $\&$ Kieff quienes reportaron una prevalencia entre un $1.4 \mathrm{y}$

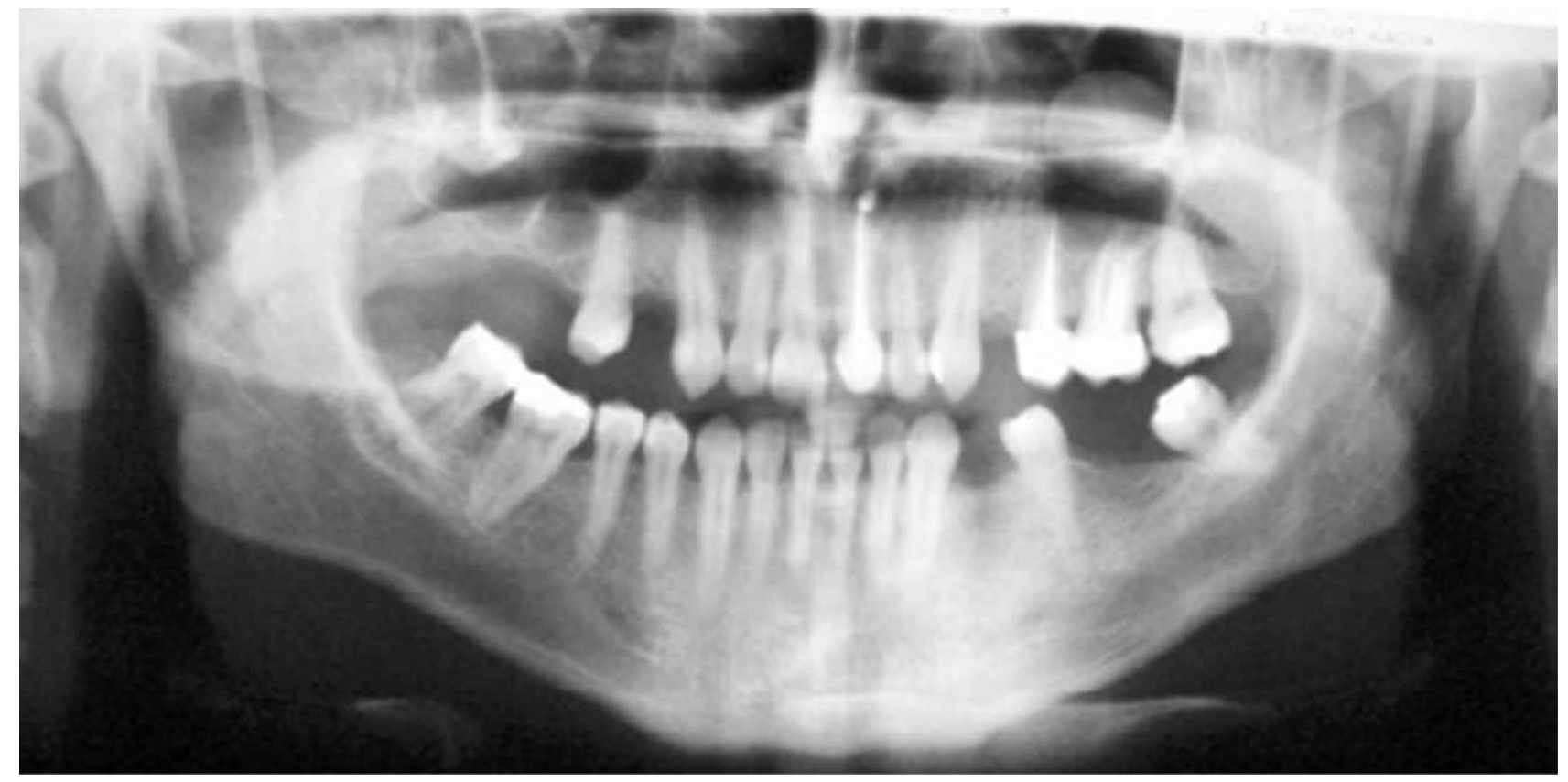

Fig.1. Quiste de retención mucoso en el seno maxilar izquierdo. 
9.6\%. Además De Oliveira et al. y Busaba \& Kieff mencionan que se observan con mayor prevalencia entre la tercera y cuarta década de vida y en el género masculino, lo que coincide con los resultados aquí presentados, ya que el promedio de edad en nuestros pacientes involucrados fue 30,1 años y en un $85.7 \%$ se presentó en hombres. Todos los casos fueron asintomáticos o por lo menos los paciente no relataron sintomatología que se pudiese asociar a estos quistes lo que concuerda con Hadar et al.; De
Oliveira et al. y Chiarini et al. quienes manifestaron que pueden presentarse asintomáticos. Radiográficamente, como lo muestra la Fig. 1. la lesión se observa, al igual que lo reportado por Casamassimo \& Lilly como una figura redondeada y radiopaca. Por lo tanto, todos los casos solo fueron pesqui-sados como hallazgos radiográficos; de ahí la relevancia de un examen radiológico panorámico. A los pacientes se les informó de su patología y fueron derivados al especialista.

FUENTES, F. R.; GARAY, C. I. \& BORIE, E. E. Presence of mucus retention cysts in maxillary sinus detected by panoramic radiographs on patients of Temuco, Chile. Int. J. Morphol., 26(3):697-699, 2008.

SUMMARY: Mucus retention cyst are a pathology with very low frequency in maxillary sinus, but easy to detect in panoramic radiographs. The aim of this study was quantify mucocele cases in maxillary sinus along five months, detecting their frequency by age and sex. It was found a prevalence of $2.06 \%$, noting more frequently in male sex and with an average age of 30 . All cases were presented without previous symptomatology.

KEY WORDS: Mucus retention cyst; Maxillary sinus; Panoramic radiographs.

\section{REFERENCIAS BIBLIOGRÁFICAS}

Allard, R.; Kwast, W. \& Van der Waal, I. Mucosal antral cysts: Review of the literature and report of a radiographic survey. Oral Surg., Oral Med, Oral Pathol., 51:2-9, 1981.

Busaba, N.Y. \& Kieff, D. Endoscopic sinus surgery for inflammatory maxillary sinus disease. Laryngoscope, 12: 1378-83, 2002.

Casamassimo, P. \& Lilly, G. Mucosal cysts of the maxillary sinus: A clinical and radiographic study. Oral Surg., Oral Med, Oral Pathol., 50:282-6, 1980.

Chiarini, L.; Nochini, P.F.; Bedogni, A.; Consolo, L.; Giannetti, L. \& Merli, A. Intracranial spread of a giant frontal mucocele: case report. Br. J. Oral Maxillofac. Surg., 38:637-40, 2000.

De Oliveira, A.; Gonzaga, A.; Narutumo, E.; Akaki, F.; Sampaio, A. \& Matsuyama, C. Mucocele frontoetmoidal: relato de casos e revisao da Literatura. Rev. Bras. Otorrinolaringol., 70(6):850-4, 2004.

Gothberg, T.; Little, J.; King, D. \& Bean, L. A clinical study of cysts arising from mucosa of the maxillary sinus. Oral Surg., Oral Med, Oral Pathol., 41:52-8. 1976.

Hadar, T.; Severo, J; Nageris, B.I. \& Yaniv, E. Mucus retention cyst of the maxillary sinus: the endoscopic approach. Br. J. Oral Maxillofac. Surg., 38:227-9, 2000.
Peric', A.; Baletic', N.; Vukomanovic'-Durdevic', B.; Jovic', M. \& Kozomara, R. Mucocoele of the maxillary sinus. Vojnosanit Pregl., 65:361-4, 2007.

Ruprecht, A.; Batniji, S. \& El-Neweihi, E. Mucous retention cyst of the maxillary sinus. Oral Surg., Oral Med, Oral Pathol., 62:728-31, 1986.

Shiomi, Y.; Shiomi, Y. \& Oda, N. Endoscopic trans-nasalvestibular approach to the maxillary sinus-application for mucoceles of the maxillary sinus. Auris Nasus Larynx, 29:65-7, 2002.

Dirección para correspondencia:

Prof. Dr. Ramón Fuentes Fernández

Depto. Odontología Integral

Universidad de La Frontera

Manuel Montt 112

Temuco-CHILE

Email: rfuentes@ufro.cl

Recibido : 14-03-2008

Aceptado: 05-06-2008 
